\title{
STUDIES OF SPACE CHARGE LOSS MECHANISMS ON THE ISIS SYNCHROTRON
}

\author{
C M Warsop, D J Adams, B G Pine, Rutherford Appleton Laboratory, Oxfordshire, UK.
}

\section{Abstract}

The ISIS Facility is the pulsed neutron and muon source based at the Rutherford Appleton Laboratory in the UK. Operation centres on the $50 \mathrm{~Hz}$ synchrotron, which accelerates $\sim 3 \times 10^{13}$ protons per pulse from 70 to $800 \mathrm{MeV}$, providing a mean power of about $0.2 \mathrm{MW}$. As commissioning of a second harmonic RF system is completed, it is expected that the main loss mechanisms will be related to transverse space charge forces, which are particularly strong during the multi-turn injection and trapping processes. Here, we describe progress in ongoing studies to understand more about what drives loss and thus limits intensity. Results from simulations and application of relevant theory are presented, concentrating on the effects thought most important for the ISIS ring. Progress on work looking at the half integer resonance and image effects in the rectangular vacuum vessels is reported, along with work for experimental studies.

\section{INTRODUCTION}

\section{ISIS Operations and Space Charge}

The ISIS ring has a mean radius of $26 \mathrm{~m}$ and accelerates about $3 \times 10^{13}$ protons per pulse (ppp) from 70 to $800 \mathrm{MeV}$ in $10 \mathrm{~ms}$. The beam is essentially unbunched during the 150 turn charge-exchange injection process, where beam is painted over the transverse acceptances ( $400 \pi \mathrm{mm} \mathrm{mr})$. Most beam loss $(\sim 5 \%)$ occurs during "trapping", when the beam is non-adiabatically bunched in the first $0.5 \mathrm{~ms}$ of acceleration. A dual harmonic RF system [1] is now operational, reducing this loss by maximising capture and improving the bunching factor. Once the optimisation of this system is complete, it is expected that the peak line density reached during trapping, and the associated transverse space charge, will impose the final limit on intensity.

This work addresses only transverse space charge. The nominal tunes of the ISIS ring are $Q_{x}=4.31, Q_{y}=3.83$; during trapping peak incoherent tune depressions are $\delta Q_{\text {incoh }} \sim 0.4$ in both planes. Main effects are expected to be the action of half integer resonances $\left(2 Q_{x}=8,2 Q_{y}=7\right)$ and image forces related to orbit errors. In this paper we report results of simulations studying these effects.

\section{HALF INTEGER RESONANCE}

\section{The Transverse Space Charge Limit}

In a previous paper [2] the main theoretical expectations for transverse space charge on ISIS, based on coherent resonance theory, were summarised. Here, simulations are used to bridge the gap between the idealised theoretical models and more realistic beams, within the 2D approximation.
The actual achievable intensity on a ring like ISIS, and its relation to the idealised incoherent and coherent limit, are of significant interest. Whilst the limitations in the (non self-consistent) incoherent picture have been well publicised [3], the limitations in the coherent approach, as applied to more realistic beams, have had limited study. When more realistic considerations are included in the coherent model, e.g. increased aperture requirements to accommodate envelope oscillations, or rms emittance $\left(\varepsilon_{r m s}\right)$ increase, the "coherent advantage" may be reduced. In this work we use 2D PIC (particle in cell) simulations, with nominal ISIS parameters, to see how near the coherent limit we might approach, and to gain some insight into the loss mechanisms.
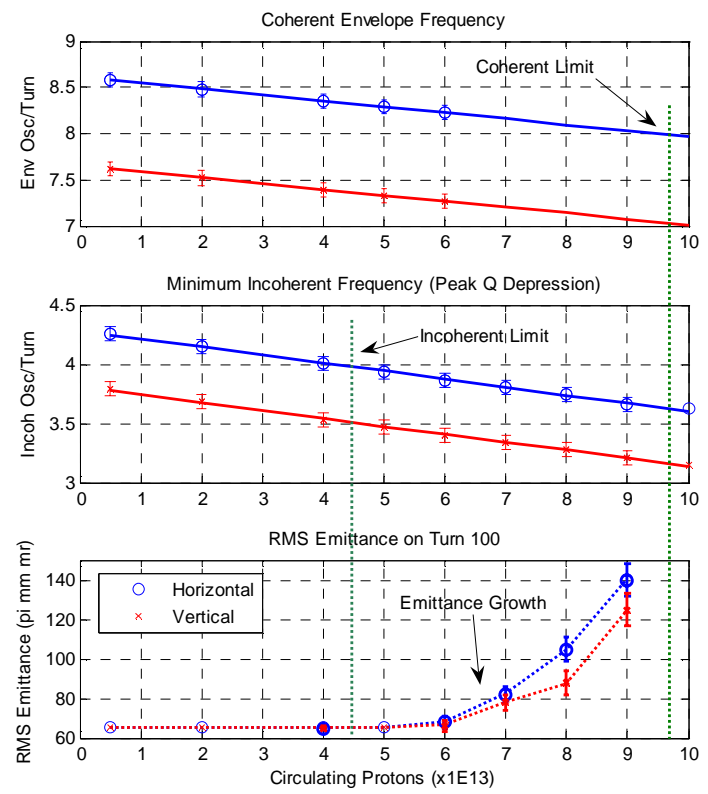

Figure 1: Coherent envelope frequency, peak incoherent tune shifts, and $\varepsilon_{r m s}$ vs intensity (from simulations).

\section{The ISIS Model and Simulations}

Simulations used the ORBIT code [4] and were also confirmed with a new "in house" code under development at ISIS. To ensure consistent and convergent results, comparisons were made between codes and for a range of key simulation parameters. Conservative choices were made: between $5 \times 10^{4}$ and $2 \times 10^{5}$ macro-particles, a $128 \times 128$ grid for particle binning and field calculations, and $>80$ space charge kicks per betatron wavelength.

Simulations used a representative, but approximate and idealised 2D model of the ISIS beam. A 4D waterbag with $\varepsilon_{x r m s} \approx \varepsilon_{y r m s} \approx 65 \pi \mathrm{mm} \mathrm{mr}$ was matched appropriately, and tracked around the ring for 100 or 1000 turns. Harmonic driving terms $\left(2 Q_{x}=8,2 Q_{y}=7\right)$ were included in the detailed lattice model to give representative half integer stop band widths $\left(\delta Q_{s b} \approx 0.02\right)$. The models excluded 
momentum spread. Simulations used the injection energy of $70 \mathrm{MeV}$ and the same nominal (zero intensity) $Q$ values. Runs were performed for a range of intensities equivalent to coasting beams of 0.5 to $9.0 \times 10^{13} \mathrm{ppp}$. Key beam parameters were recorded, with most values derived from the standard ORBIT functions (but also evaluated independently by the ISIS code). To assess the importance of mismatch two sets of runs were performed: "small" and "large" mismatch, corresponding to initial $\sim 5 \%$ and $\sim 25 \%$ oscillations in rms widths respectively.

\section{Overall Results and Matching Effects}

Overall results are summarised in Figure 1. This shows coherent envelope tunes, peak incoherent tune depressions and $\varepsilon_{r m s}$ as a function of intensity. Significant beam growth appears between the incoherent and coherent limits. Studies of matching are summarised in Figure 2: these show evolution of $\varepsilon_{r m s}$ and oscillations of envelope width and angle over 100 turns, for the small and large mismatch at 4,6 and $8 \times 10^{13} \mathrm{ppp}$. The effect of mismatch is also indicated by error bars on the $\varepsilon_{r m s}$ in Figure 1. At low intensity, $\varepsilon_{r m s}$ is reasonably preserved regardless of match. At medium intensity, there is clearly more growth in the mismatched case, although the increase in $\varepsilon_{r m s}$ is moderate. At high intensity the envelope resonance dominates, and initial match has a small effect.
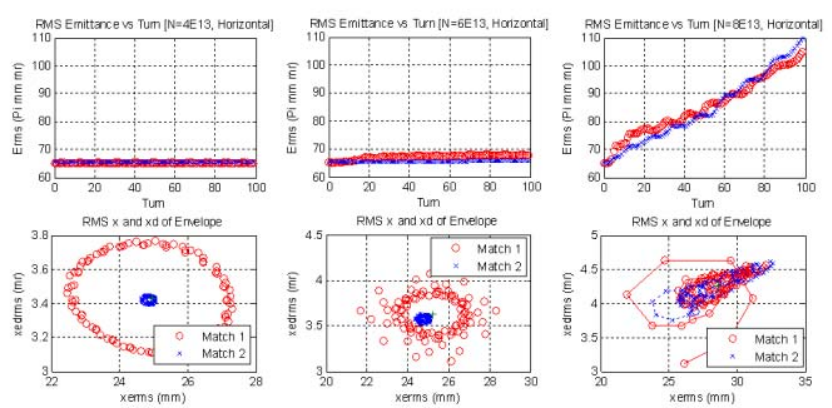

Figure 2: Evolution of RMS emittance (top) and RMS envelope (bottom) for small (blue) and large (red) mismatch. Intensities are $4,6,8 \times 10^{13}$ from left to right (horizontal).

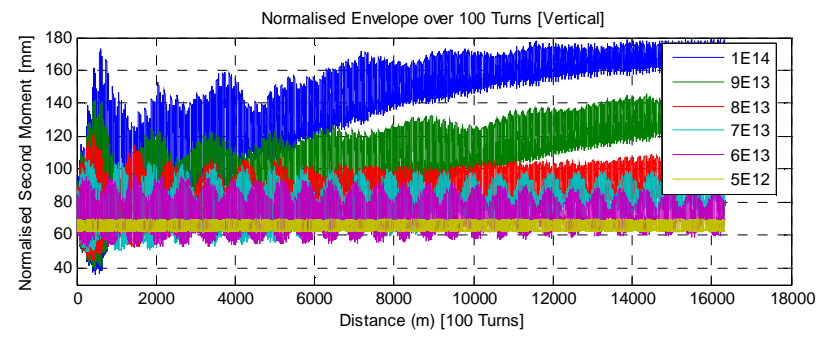

Figure 3: Evolution of Envelopes over 100 turns.

Evolution of envelopes for a range of intensities (large mismatch) are shown in Figure 3. At low intensities, motion is characterised by regular, moderate beating, consistent with a conserved $\varepsilon_{r m s}$. As intensity increases, the oscillations become larger, eventually resulting in net growth of envelope and $\varepsilon_{r m s}$.

\section{Phase Space Distributions, Emittance Growth}

The development of particle phase space distributions is shown in Figures 4, 5 and 6. Only one transverse plane is shown (horizontal), as planes are equivalent. Coordinates are normalised: horizontal axis is displacement $x(\mathrm{~mm})$, vertical axis is normalised angle $(\alpha / \beta) x+x^{\prime}(\mathrm{mr})$, with $x^{\prime}$ the angle, and $\alpha, \beta$ the lattice Twiss parameters [4].

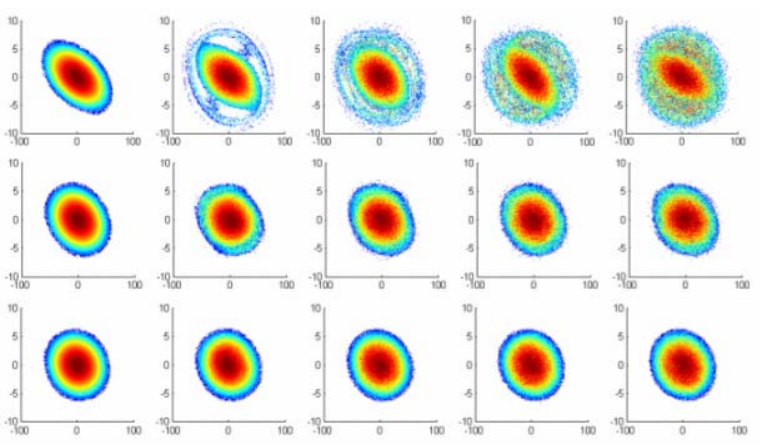

Figure 4: Evolution of normalised transverse distribution (horizontal) for small mismatch ( $\sim 5 \%$ in rms width). Plots show turns 1, 21, 41, 61, 81 from left to right and intensities $4,6,8 \times 10^{13} \mathrm{ppp}$ from bottom to top.
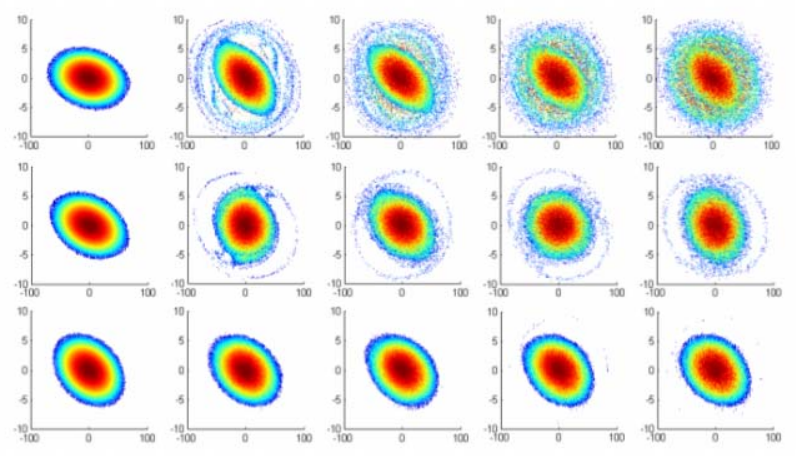

Figure 5: Evolution of normalised transverse distribution for large mismatch ( $25 \%$ in rms width), otherwise as above.
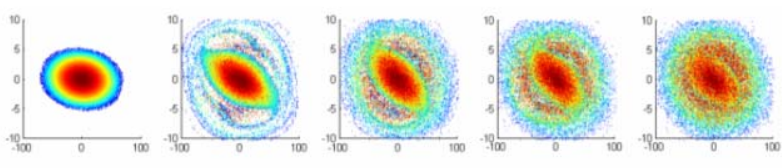

Figure 6: Evolution of normalised transverse distribution near resonance. As above, but at $9 \times 10^{13} \mathrm{ppp}$.

In these figures, particles are labelled with the same colour over multiple turns (from left to right), corresponding roughly to their emittance on the first turn. Dark red particles start in the "inner-core", and blue particles in the "outer-core". This gives an indication of where halo particles originated. Comparing Figures 4 and 5 shows that a large mismatch generates more halo. However, at high intensities as envelope resonance is approached, mismatch is a minor effect as the beam rapidly blows up, as shown in Figure 6. 
The above results are all for $\leq 100$ turns; Figure 7 shows evolution of $\varepsilon_{r m s}$ over 1000 turns. Error bars indicate estimated numerical uncertainties.

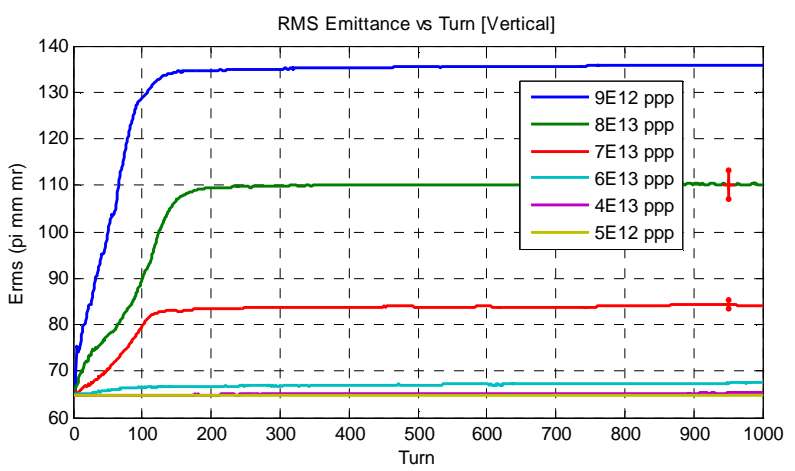

Figure 7: Evolution of $\varepsilon_{r m s}$ over 1000 turns as a function of intensity.

\section{Interpretation of Results}

Taken at face value, the results suggest that with the magnet errors and mismatch levels expected on ISIS, significant beam growth will occur at $6-8 \times 10^{13} \mathrm{ppp}$ for a coasting $70 \mathrm{MeV}$ beam. Using simple scaling for bunching factor and energy, this suggests operational limits of around $2.1-2.8 \times 10^{13}$ and $2.8-3.7 \times 10^{13} \mathrm{ppp}$ for single and dual harmonic $\mathrm{RF}$ respectively (optimisation of bunching factor may well increase the latter).

Results indicate that the main emittance growth does not occur at the incoherent limit. In addition, the lack of "inner-core" particles appearing in the halo (Figures 4, 5) at low and medium intensities supports the expectation that incoherent resonance is not the main (initial) loss mechanism: the "outer-core" particles are lost first. Phase space structure indicates parametric particle resonance driven by the envelope oscillations (as the envelope approaches resonance). At lower intensities, ideas of the coherent model seem to describe behaviour well, with the expected regular beating of envelopes and expected tune depressions. At about $7 \times 10^{13}$ ppp behaviour changes markedly: envelopes and $\varepsilon_{r m s}$ indicate substantial growth. Figure 7 shows this clearly, also indicating that the beam redistributes from the non-stationary waterbag to a new stable distribution within about 200 turns.

Further work is planned to understand these results, associated loss mechanisms and possible code limitations. Although some care has been taken to ensure that results were convergent and consistent, the possibility of nonphysical effects remains (particularly with 1000 turn tracking and halo). Ultimately, only comparison with experiment and other algorithms will confirm the results. It is also possible that the simplified 2D beam model will behave quite differently from the real beam.

\section{IMAGE EFFECTS}

\section{Initial Calculations with Images}

When the possibility of closed orbit errors (off-axis beams) and the ISIS rectangular vacuum vessels are included, additional "image" driving terms appear [3].
The code under development at ISIS includes a field solver with boundary conditions appropriate for the ISIS vacuum vessels (which has been benchmarked against CST-Studio ${ }^{\circledR}[5]$ ): Figure 8. Figure 9 shows results from some initial tests comparing coherent dipole tune shifts from simulations for the "direct" and "image" field solvers. These show the expected results, with coherent dipole shift only appearing with images. This will allow study of image related losses on ISIS.

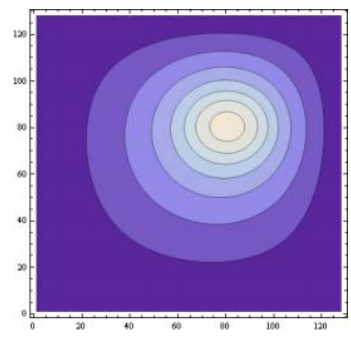

Figure 8: Example of space charge field solver: potential of off centre waterbag beam in rectangular vessel.

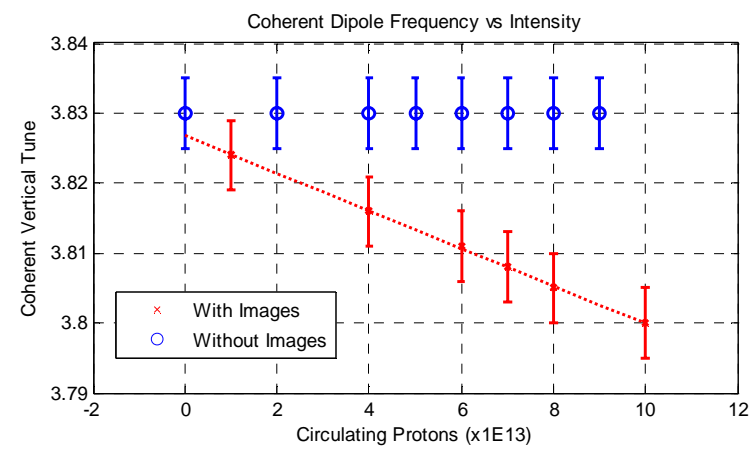

Figure 9: Example of coherent dipole Q shifts

\section{FUTURE AND RELATED WORK}

A key aim of this work is to compare theory and simulation with experimental results from ISIS. Diagnostics and experimental methods are being developed to this end, including new profile monitors [6] and procedures for storage ring mode experiments. Simulations will next look at longitudinal effects. Related studies on ISIS injection are reported in [7].

\section{REFERENCES}

[1] A Seville et al, "Progress on Dual Harmonic Acceleration on the ISIS Synchrotron", Proc. PAC07

[2] C M Warsop, "Transverse Space Charge Studies for the ISIS Synchrotron", Proc. EPAC06.

[3] R Baartman, "Betatron Resonances with Space Charge", AIP Conf. Proc. CP448, 1998.

[4] J A Holmes et al, ORBIT User Manual, ORNL, Technical Note SNS/ORNL/AP/011

[5] www.cst.com

[6] S J Payne et al, "A Self Calibrating Real Time MultiChannel Profile Monitor for the ISIS Proton Synchrotron”, Proc. DIPAC07

[7] B Jones et al, "Injection Studies on the ISIS Synchrotron", Proc. PAC07 